\title{
Evaluating the Effectiveness of Short-Term Treatment at a University Counseling Center
}

\author{
M. Elizabeth Vonk, Ph.D. \\ Ph.D., University of Georgia, 1996 \\ Clinical Social Worker, \\ Emory University Counseling Center, Atlanta, Georgia
}

\section{Statement of the Research Problem}

In this study, the effectiveness of short-term treatment in reducing the psychosocial symptomatology of university counseling center clients was examined within the context of an overall program evaluation. Evaluation of practice is an essential component of social work practice in counseling centers just as it is in other settings.

Students who utilize counseling centers for clinical treatment present with a wide variety of problems including, but not limited to interpersonal difficulties, academic concerns, eating disorders, and victimization from abuse. The impact of these problems on the client is made more clear by examining the symptoms that clients are experiencing. Regardless of the presenting problems, the presenting psychosocial symptoms generally involve the areas of depression and anxiety. Distress related to psychosocial symptomatology has been found to be quite high among counseling center clients, with up to approximately $30 \%$ of students meeting the criteria for psychiatric disturbance at the time of initial contact (Johnson, Ellison, \& Heikkinen, 1989). A student who is experiencing a high level of psychosocial symptomatology is not able to function at his or her best in the academic setting, making the reduction of that level an important goal for the social worker and client.

The level of disturbance among counseling center clients, combined with an increasing demand for clinical service, creates a challenge to which counseling centers must respond. One common response has been the imposition of limits on the allowable number of counseling sessions for each client. Thus, short-term counseling has become the primary treatment model at many counseling centers. Yet rather than being a specific model of treatment that is theoretically derived, short-term counseling has been administratively driven and varies among centers and counselors in focus, duration, and therapeutic characteristics (Burlingame \& Fuhriman, 1987)

Reviews of relevant literature have led some to conclude that there is little evidence that particular clients are more effectively treated in long-term versus short-term treatment (Bloom, 1992; Budman \& Gurman, 1983). Others (Gelso \& Johnson, 1983) suggest that clients who have chronic, severe problems may be more effectively treated in time-unlimited treatment. Based on their research findings and previous literature, they speculate that this is due in large part to the difficulty in quickly establishing a therapeutic alliance with this type of client rather than to the 
level of his or her disturbance. Even with this caveat, many counselors have reservations about short-term treatment and expect poorer results than are warranted.

Along with the challenge of meeting the rising clinical demand, accountability for the services that are provided by counseling center staff has become increasingly important. College and university budgets have become tighter, leading to more competition for funding. Both clients and staff members are also concerned with the quality of service. Social workers and others who staff counseling centers must provide evidence of the scope and effectiveness of their work in every area of service including clinical treatment. Since short-term, non-specific counseling is so widely used, it is crucial that its effectiveness be evaluated; indeed, the evaluation of effectiveness should be part of a counseling center's overall program evaluation.

In spite of this growing need, there are very few published empirical program evaluations of student counseling centers. Of the small number of evaluations that are available, only four have been identified that are focused specifically on short-term treatment evaluation. The earliest (Harmon, 1971) employed a post-test only design and relied on a standardized measure of client satisfaction. Gelso, Spiegel, \& Mills (1983) examined treatment effectiveness along with comparing clients' and counselors' reactions to short-term treatment through the use of locally developed instruments with limited evidence of reliability and validity that measured the perception of change and satisfaction with counseling. In both cases, the clients' change from preto post-treatment was assessed only through client and counselor perception rather than through more objective repeated measures. Keilson, Dworkin, \& Gelso (1983) used a standardized instrument at pre- and post-treatment to examine effectiveness. The main disadvantage of their study was the exclusion of clients who were outside the normal to moderately disturbed range. Related to this, they used an instrument that was appropriate for their sample, but inappropriate for use with more severely disturbed clients who are being treated at counseling centers in increasing numbers. Turner, Valtierra, Talken, Miller \& DeAnda (1996) administered the MMPI2 , the College Adjustment Scale and the Client Satisfaction Questionnaire to 94 college students before randomly assigning them to either 30-minute session or 50-minute session therapy. After completing a maximum of eight sessions, the assessments were completed again. Both groups improved equivalently, and adjustment was apparently independent of treatment session length. Only one of these four prior studies used a standardized general measure of psychosocial symptoms that would be applicable to diverse clients in the context of a pre-posttest research design. (Tumer et al., 1996), thus there is a clear need for further examination of the effectiveness of short-term treatment in counseling centers.

\section{Research Ouestions}

The present study represents an effort on the part of the Counseling Center (CC) staff to improve the evaluation of treatment effectiveness through the use of a standardized instrument. In broad terms, we wanted to determine if psychosocial treatment at the $\mathrm{CC}$ was effective. Due 
to the preponderance of individual, non-specific short-term treatment at the CC, as opposed to other treatment methods such as group or couple counseling, the focus of this evaluation was on the former. Treatment was considered to be effective if the recipient showed significant improvement (i.e., a decline in psychosocial symptomatology). More specifically, the independent variable, short-term treatment referred to any individual treatment administered by a $\mathrm{CC}$ staff member for a minimum of four and maximum of 20 sessions. The dependent variable, psychosocial symptomatology was defined by the items on the outcome measure. One hypothesis was tested:

clients who receive short-term treatment at the $\mathrm{CC}$ will show a decrease in psychosocial symptomatology.

\section{Methodology}

Emory University, located in Atlanta, Georgia, is a private institution which enrolls approximately 10,400 undergraduate, graduate, and professional students, all of whom are served by the University Counseling Center. The Counseling Center staff consists of licensed psychologists, licensed social workers, and interns from both fields. Staff members represented diverse theoretical orientations.

This study utilized a quasi-experimental, nonequivalent control group design (Royse \& Thyer, 1996) with additional testing of the control group following delayed treatment. Although there was no random assignment, this design allowed for a comparison between the immediate treatment group and a no-treatment, wait-list group. In addition, the group who waited received delayed treatment. The design did not eliminate all alternative explanations for change, but was sufficient to answer the question of whether clients who received short-term, individual treatment at the $\mathrm{CC}$ showed a decrease in symptomatology when compared to similar clients receiving no treatment.

The outcome measure that was chosen to measure psychosocial symptomatology is the Symptom CheckList-90-Revised (SCL-90-R) (Derogatis, 1992). The SCL-90-R is a widely used, psychometrically sound, 90 item, self-report instrument that measures the perception of the presence of psychosocial symptoms during the past seven days. Nine symptom dimensions are measured with the SCL-90-R along with a global severity index (GSI). It also includes a simple method to determine whether a profile is positive or negative for the presence of psychiatric disorder.

For this project, a purposive sample was used, made up of those clients who met the following criteria: 
1) began treatment after August 21, 1995 and terminated before May 10, 1996;

2) participated in a minimum of four and maximum of 20 sessions of individual treatment;

3) participated in a planned termination from treatment;

4) agreed to complete the instrument at intake and termination.

The delayed treatment group was a purposive sample who met the criteria above in addition to the following:

1) wait between intake and first treatment session totaled 21 or more days;

2) agreed to complete the instrument immediately before the first treatment session.

The sample of 55 clients represented $82 \%$ of those who were eligible for the study. Clients in the Immediate treatment group $(n=41)$ were tested both at intake (Time 1) and after treatment (Time 2). Clients in the Wait/Delayed treatment group $(n=14)$ were tested at intake (Time 1), after waiting a minimum of 21 days for treatment (Time 2), and after delayed treatment (Time 3).

Statistical analyses were conducted with SPSS/PC+(Version 5.0) (SPSS, Inc., 1992). Chi-square tests were used to compare groups by demographic variables. A correlation matrix of the nine subscale scores at Time 1 showed that 29 of the 36 relationships were significantly positive (r's ranging from .38 to $.79 ; p<.01$ ). Therefore, a $2 \times 2$ multivariate analysis of variance for repeated measures was used in order to adjust for correlated dependent variables when comparing means for the nine subscales. The GSI was considered separately using a $2 \times 2$ ANOVA for repeated measures because it is a global score that takes all of the subscale scores into account. Post-hoc Scheffe' tests were used following significant interaction effects to determine the location of differences.

Results

Data analyses indicate that demographic variables and symptomatology were similar for the two groups at Time 1 . There was a statistically significant decrease in symptomatology from Time 1 to Time 2 in the Immediate treatment group as compared to the Wait/Delayed treatment group for the Global Severity Index (GSI) and for all but one subscale. Symptomatology also showed a statistically significant decrease from Time 2 to Time 3 in the Wait/Delayed treatment group for the GSI. A change score for the GSI was computed and examined in relationship to the 
number of sessions and found to be nonsignificant. In addition, while the proportions of clients meeting the SCL-90-R criteria for a psychiatric disorder were statistically similar at Time 1 , analysis revealed that the immediate treatment group had a statistically significantly smaller proportion of positive cases at Time 2 .

\section{Utility for Social Work Practice}

The data are supportive of the short-term treatment effectiveness at the $\mathrm{CC}$ and add support to previous studies that found short-term treatment at counseling centers to be effective. This study makes contributions on three levels. First, it provides valuable information to the staff, clients, and administration of the $\mathrm{CC}$ concerning the effectiveness of the treatment that they are providing. Administrators benefit from the results of this study which provide justification for continued financial support of the counseling center. Staff members benefit from the knowledge that the clinical service that is being provided by them is effective. They also have more objective information available to them on which to base policy decisions such as maximum allowable sessions. Finally, if policy change allows for more clients to be seen at the counseling center rather than being referred, clients will benefit as well.

Next, the study provides an example of agency based practice-evaluation carried out through the leadership of a social worker. Directly applicable to social workers in counseling center settings, it also has relevance for social workers in a number of other agencies in which short-term treatment has become the norm. Along with the benefits to the center mentioned above, this study shows that the constraints of the agency setting do not make research impossible or without value. Quasi-experimental designs in such real-life settings can be practical and help to control for some alternative explanations of change. Also, the use of unspecified short-term treatment as the independent variable provides a closer approximation to "real life" counseling than studies that utilize a specific short-term model or protocol-driven treatment (Budman \& Gurman, 1983). The use of a generally atheoretical measure of symptomatology such as the SCL-90-R can be applied by clinicians using diverse treatment models. The choice of methodology and standardized measure ensure that it can easily be replicated at other counseling centers or agencies.

Finally, this study adds to what is known about the effectiveness of short-term treatment in counseling centers. It differs from the previous ones in several ways. First, unlike Harmon (1971) and Gelso et al. (1983) who assessed change due to treatment through client and counselor perception only after counseling had ended, this study utilized a standardized objective measure of psychological symptomatology both before and immediately after treatment. Next, while Keilson et al. (1983) used standardized measures with a pretest-posttest design, they did not include severely disturbed clients. In the present study, the majority $(71 \%)$ of clients met the SCL-90-R criteria for a psychiatric disorder at pre-test, thus providing support for the effectiveness of short-term treatment with more severely disturbed students. These results also 
provide some support for the conclusion reached both by Budman \& Gurman (1983) and Bloom (1992) that there is little evidence that clients with severe levels of psychopathology are more effectively treated in long-term rather than short-term therapy. As Gelso \& Johnson (1983) suggest, however, there may be factors other than level of disturbance, such as interpersonal problems that interfere with the therapeutic alliance, that indicate the use of more long-term treatment. Unlike Turner et al. (1996), the present study included a no-treatment waiting list period with assessments conducted at entry into, and termination from, the waiting list, allowing for some control over the variable of spontaneous remission. In short, the results of preand posttesting clients of all levels of psychiatric disturbance using a standardized objective measure add an important piece of outcome information that has been missing to this point. 
References

Bloom, B.L. (1992). Planned short-term psychotherapy. Needham Heights, MA: Allyn and Bacon.

Budman, S.H. \& Gurman, A.S. (1983). The practice of brief therapy. Professional Psychology: Research and Practice, 14, 277-292.

Burlingame, G. M. \& Fuhriman, A. (1987). Conceptualizing short-term treatment: A comparative review. The Counseling Psychologist, 15, 557-595.

Derogatis, L. R. (1992). SCL-90-R administration, scoring \& procedures manual-II. Towson, MD: Clinical Psychometric Research, Inc.

Gelso, C. J. \& Johnson, D. H. (1983) Explorations in time-limited counseling and psychotherapy. New York: Teachers College, Columbia University.

Gelso, C.J., Spiegel, S.B., \& Mills, D.H. (1983) Clients' and counselors' reactions to timelimited and time-unlimited counseling. In C.J. Gelso and D.H. Johnson (Eds.), Explorations in time-limited counseling and psychotherapy (pp. 14-62). New York: Teachers College, Columbia University.

Harman, R. L. (1971). Client assessment of a university counseling service. Joumal of Counseling Bsychology, 18, 495-497.

Johnson, R. W., Ellison, R. A., \& Heikkinen, C. A. (1989). Psychological symptoms of counseling center clients. Journal of Counseling Psychology, $36,110-114$.

Keilson, M.V., Dworkin, F.H., \& Gelso, C.J. (1983). The effectiveness of time-limited therapy in a university counseling center. In C.J. Gelso and D.H. Johnson (Eds.) Explorations in time-limited counseling and psychotherapy (pp. 5-13). New York: Teacher's College, Columbia University.

Royse, D., \& Thyer, B. A. (1996). Program evaluation: An Introduction. (2nd edition) Chicago: Nelson-Hall Publishers.

SPSS/PC+(5.0) [Computer software]. (1992). Chicago: SPSS, Inc.

Turner, P.R., Valtierra, M., Talken, T.R., Miller, V.I., \& DeAnda, J. (1996). Effect of session length on treatment outcome for college students in brief therapy. Journal of Counseling Psychology, 43, 228-232. 